\title{
Les nouveaux concepts de réacteurs nucléaires
}

Annick Billebaud, physicienne, CNRS

\author{
Les recherches sur des nouveaux types de réacteurs nucléaires pouvant succéder aux réacteurs \\ actuels sont des travaux de longue haleine. De nombreux nouveaux concepts sont à l'étude \\ mais, depuis 2000 , un forum international incite la recherche à se concentrer sur quelques \\ systèmes prometteurs vis-à-vis des nouveaux critères que devraient remplir des réacteurs \\ de $4^{\mathrm{e}}$ génération. En France, les systèmes étudiés dans ce cadre sont les réacteurs à neutrons \\ rapides refroidis au sodium et les réacteurs à sels fondus. Les réacteurs pilotés par accélérateur, \\ s'inscrivant dans une stratégie d'incinération de déchets dans des systèmes dédiés, \\ font également l'objet d'études depuis vingt ans.
}

\section{Pourquoi étudier de nouveaux systèmes ?}

Dans un cœur de réacteur nucléaire, les réactions à l'œuvre conduisant finalement à la production d'énergie thermique, et en particulier les fissions, sont bien connues et communes à tous les réacteurs. Cependant, les façons d'exploiter la réaction en chaine, de la contrôler, de consommer le combustible, ou d'extraire la chaleur du réacteur peuvent être réalisées de différentes manières satisfaisant divers critères prioritaires que nous verrons plus loin.

Or, un nouveau concept, différant de ceux qui ont existé ou sont en cours d'exploitation, prend du temps à être démontré et validé ; il nécessite des travaux de modélisation et, à un moment ou à un autre, des expériences sur maquette puis la construction de prototypes pour étayer sa faisabilité. Il faut bien souvent plusieurs décennies de travail de recherche, de conception, de développement, d'expérimentation pour aboutir à un système prêt à passer à l'échelle industrielle, ce qui dépasse souvent la durée de la carrière professionnelle d'un individu. Cette échelle de temps est la raison principale pour laquelle les organismes de recherche scientifique et technologique tentent d'anticiper les besoins futurs. Dans le cas du nucléaire, cela implique de revisiter d'anciens concepts de réacteurs ou d'en proposer de nouveaux à la lumière des dernières connaissances et avancées. La France est un pays qui a de fortes compétences dans les sciences nucléaires et les technologies associées aux réacteurs, et possède donc un creuset favorable à ce type de recherches. L'objectif est de pouvoir disposer, au-delà de 2030 (voire 2040 ou 2050 pour les plus innovantes), de solutions ayant démontré leur faisabilité. Cette recherche explore et prépare de possibles solutions dans son champ de compétences, et ne présage pas des choix futurs qui reviennent aux politiques et à la société. Néanmoins, pour les mêmes raisons d'inertie temporelle, les choix des axes de recherche engagent quelque peu l'avenir.

\section{Qu'est-ce que \\ la " génération IV » ?}

Les réacteurs nucléaires en cours d'exploitation en France font partie de ce qui est appelé la deuxième génération. On considère que les versions améliorées en cours de construction, comme l'EPR, sont la troisième génération. Dans de nombreux pays, des recherches prospectives sont menées pour imaginer des réacteurs de quatrième génération tournés vers le futur. Sous l'impulsion du Département de l'Énergie des États-Unis, un forum international, le GIF (en anglais, Generation IV International Forum, regroupant une douzaine de pays dont la France) s'est formé en 2000. Son objectif est d'encourager les recherches à l'échelle internationale sur quelques concepts de réacteurs qui rempliraient des critères spécifiques, et dont la mise en œuvre éventuelle se ferait à l'horizon 2030-2040. Ces critères sont définis comme des objectifs d'amélioration nécessaires sur les plans économique, environnemental et social, si l'énergie nucléaire devait contribuer notablement à répondre à la demande énergétique mondiale du vingt-et-unième siècle. Ils portent sur quatre domaines principaux.

- Durabilité : ce critère a pour but de pouvoir inscrire l'énergie nucléaire dans le temps, en optimisant à la fois les ressources en combustible et la production de déchets, et donc leur gestion à long terme. En effet, les systèmes actuels sont essentiellement basés sur l'utilisation de combustible enrichi en uranium 235, ce qui nécessite de traiter de grandes quantités d'uranium pour extraire cet isotope ; il faut environ 200 tonnes d'uranium chaque année pour faire fonctionner un cœur de réacteur à eau sous pression de $1 \mathrm{GW}$ qui fera fissionner seulement une tonne de matière. Dans le futur, on souhaite pouvoir utiliser un système qui permette d'utiliser tout le potentiel du minerai extrait et d'envisager ainsi plusieurs siècles de production d'énergie à l'échelle mondiale.

- Sureté et fiabilité : il s'agit ici, d'une part, de minimiser les risques d'accidents 


\section{$\gg>$}

nucléaires conduisant entre autres à des catastrophes comme celles de Tchernobyl ou Fukushima, et d'autre part, dans l'éventualité où un accident se produirait, de minimiser l'impact sur les populations et l'environnement. Pour cela, l'accent est mis sur la sureté passive des systèmes et en particulier l'évacuation de la puissance résiduelle en cas d'arrêt du cœur.

- Compétitivité économique : dans un marché de l'énergie libéralisé où les sources de production sont de plus en plus diverses, un nouveau système doit pouvoir être compétitif.

- Résistance à la prolifération et protection physique : le détournement des installations et des matières nucléaires civiles à des fins militaires est un risque depuis longtemps sous haute surveillance internationale. À ce risque s' ajoute aujourd'hui celui d'actes malveillants, voire terroristes. Par conséquent, il est proposé d'intégrer dès la conception des nouveaux systèmes une protection contre le vol de matières radioactives et les actes de sabotage qui pourraient intervenir dans les installations ou les transports de matière.

\section{Quels systèmes pour répondre à ces critères ?}

Un réacteur nucléaire se définit par rapport à ses trois composantes principales que sont : le combustible, non seulement sa nature mais aussi sa géométrie, sa composition, sa forme chimique ; le modérateur, matériau présent dans le cœur permettant d'optimiser l'utilisation du combustible en ralentissant plus ou moins les neutrons ; et le caloporteur, qui sert à transférer la puissance thermique du cœur vers les échangeurs pour la transformer en électricité. Pour la plupart des réacteurs actuels, l'eau joue à la fois le rôle de modérateur et de caloporteur. Il existe un nombre très élevé de combinaisons possibles à partir de ces trois éléments, et donc de nombreuses variantes de réacteurs nucléaires potentiels. Au cours de l'histoire du nucléaire, quelques centaines ont été étudiées sur le plan théorique mais, au total, moins d'une vingtaine ont fait l'objet d'une construction permettant la production de puissance. Pour répondre aux nouveaux critères les possibilités restent nombreuses. Le GIF a procédé à une sélection de six concepts jugés les plus prometteurs, sur lesquels concentrer les recherches.
Trois de ces concepts sont à neutrons dits " rapides ", c'est-à-dire conservant autant que possible l'énergie des neutrons telle qu'à leur création. Ceci a plusieurs avantages vis-à-vis du critère de durabilité. En effet, afin d'optimiser l'utilisation du minerai d'uranium, il est possible d'envisager la régénération du combustible, en transformant les noyaux d'uranium 238 dits "fertiles " en noyaux de plutonium 239 "fissiles » ${ }^{(a)}$. Si chaque noyau d'uranium 238 produit un noyau de plutonium 239 par capture d'un neutron, on utilise ainsi tout le potentiel énergétique du minerai d'uranium, composé à $99,3 \%$ d'uranium 238. Les neutrons rapides permettent de minimiser les captures parasites de neutrons et ainsi de maintenir la criticité du système et la régénération du plutonium.

Ces réacteurs à neutrons rapides peuvent utiliser différents fluides modérateurs et caloporteurs ; les trois concepts étudiés sont le réacteur rapide au sodium (Sodium Fast Reactor, SFR), le réacteur rapide au plomb (Lead Fast Reactor, LFR) et le réacteur rapide à gaz (Gas Fast Reactor, GFR). Ils présentent tous une bonne efficacité thermique. Le sodium combine une température de fusion basse avec un point d'ébullition très élevé, ce qui offre une bonne inertie thermique au circuit primaire. Cependant, le SFR et le LFR doivent faire face aux propriétés chimiques d'un métal liquide : réactivité vis-à-vis de l'eau et de l'air pour le sodium, corrosion pour le plomb. Le troisième concept a l'avantage d'utiliser un gaz chimiquement inerte, l'hélium ; en revanche, il a de ce fait une assez faible inertie thermique en cas d'arrêt de la circulation forcée : cela n'est pas optimal pour la sureté, et nécessite le développement d'un combustible très particulier.

Le concept de réacteur à très haute température (Very High Temperature Reactor, VHTR) fonctionne entre 800 et $1000{ }^{\circ} \mathrm{C}$. Il utilise des neutrons plus lents, dits "thermiques". Il est lui aussi refroidi à l'hélium, mais modéré avec du graphite. Son avantage principal est que le gaz peut être directement utilisé dans une turbine sur le circuit primaire. La chaleur produite par ces systèmes peut également être utilisée de différentes manières sur le plan industriel, notamment pour la production d'hydrogène.

Un autre concept, revisitant celui des réacteurs à eau, est également étudié : le réacteur à eau supercritique (SuperCritical
Water cooled Reactor, SCWR) qui fonctionne avec une température et une pression situées au-dessus de celles du point critique de l'eau $\left(374{ }^{\circ} \mathrm{C}, 221\right.$ bars), d'où son nom ; il peut être conçu pour des neutrons soit thermiques, soit rapides. La vapeur produite, extrêmement chaude, peut être envoyée directement dans la turbine et, après condensation, l'eau est renvoyée dans le cœur. Ce procédé bénéficie de la longue expérience des centrales thermiques à combustible fossile utilisant l'eau supercritique. Il a un avantage de rentabilité économique car son efficacité thermique peut s'élever jusqu'à $44 \%$, à comparer aux $33 \%$ des réacteurs à eau sous pression actuels. Cependant, des défis technologiques restent à relever, comme : la modélisation des transferts de chaleur lors des accidents, en cas de dépressurisation et de perte des conditions supercritiques ; la qualification des matériaux aux hautes températures, et notamment des aciers pour les gaines du combustible ; et la démonstration de la sureté passive du système.

Le sixième concept, un des plus innovants, utilise les sels fondus (Molten Salt Reactor, MSR) : il est est présenté plus loin.

\section{Les systèmes de quatrième génération étudiés en France}

Sur la base d'estimation de ressources limitées en uranium, et d'une utilisation importante du nucléaire au niveau mondial, la France avait imaginé dès les années 1960 une stratégie qui commençait par l'exploitation des réacteurs thermiques à uranium enrichi. L'idée était de constituer un stock de plutonium (noyaux produits pendant le fonctionnement), permettant ensuite d'alimenter des réacteurs à neutrons rapides surgénérateurs et d'assurer une production d'énergie pérenne. La France a ainsi très tôt concentré ses efforts de recherche sur les réacteurs à neutrons rapides, et a fait le choix technique du sodium liquide comme caloporteur, portant le concept jusqu'à sa réalisation. Cependant, seules deux unités au sodium liquide ont été construites à l'échelle industrielle en France : Phénix (1973-2010), puis Superphénix, arrêté prématurément en 1997 pour des raisons industrielles, économiques, et politiques, associées à un contexte post-Tchernobyl. Ce type de réacteur fait à nouveau l'objet 
de recherches dans le cadre de la quatrième génération, en raison de ses qualités indéniables vis-à-vis des critères de ressources et de déchets, et avec l'avantage de reposer sur une technologie déjà mise en œuvre dans plusieurs pays. Ainsi le CEA, EDF et Framatome ont consacré d'importants efforts de recherche pour revisiter ce concept, avec notamment le projet d'un réacteur au sodium de démonstration, de $600 \mathrm{MW}$, ASTRID.

Le concept de réacteur à sels fondus (MSR) a été étudié aux États-Unis à partir des années 1950 puis abandonné dans les années 1970. Dans ce système, le combustible est dissous dans un sel fluoré liquide, qui joue ainsi aussi le rôle de caloporteur et peut circuler directement dans les échangeurs de chaleur. Réacteur modéré par du graphite dans ses premières versions, il a été revisité récemment comme un réacteur à neutrons rapides par des équipes de recherche académique françaises, notamment pour le rendre régénérateur avec un retraitement en ligne simplifié, en utilisant un combustible thorium et uranium. Le combustible sous forme de sels fondus présente de nombreux avantages : d'une part, grâce aux bonnes propriétés thermodynamiques du sel : température d'ébullition élevée, bonnes capacité calorifique et conductivité thermique ; et d'autre part, parce que, en cas de dysfonctionnement du système, le sel liquide peut être reconfiguré de façon passive : par exemple dispersé dans un réseau de réservoirs dimensionnés pour tenir de hautes températures, permettant différentes options pour évacuer sa puissance résiduelle. La possibilité de retraiter le sel en ligne permet de maintenir dans le cœur un bilan neutronique favorable à l'utilisation de différents combustibles. En totale rupture technologique avec les réacteurs existants et bien éprouvés, il demande une relecture complète de l'approche de sureté, et un considérable effort de recherche pour lever les verrous d'abord scientifiques, puis technologiques, pour tout ce qui a trait au sel et aux matériaux (corrosion, retraitement, physico-chimie, ...), et à la neutronique d'un combustible en mouvement (combustiblecaloporteur, pilotage de la criticité), autant d'étapes avant un prototype industriel. L'inertie naturelle des filières nucléaires est un frein au déploiement de ces technologies très innovantes, même si elles représentent potentiellement des solutions intéressantes pour le futur.

\section{Les réacteurs pilotés par accélérateur}

Sans faire partie des systèmes de quatrième génération étudiés par le GIF, les réacteurs pilotés par accélérateur (Accelerator Driven System, ADS) font également l'objet d'intenses études de faisabilité en raison de leur potentiel dans un scénario où l'on souhaiterait incinérer, dans des systèmes dédiés, certains déchets nucléaires produits par les filières actuelles : ceci permettrait de réduire leur radiotoxicité et leur chaleur résiduelle et par conséquent les surfaces de stockage. Ces réacteurs ont la particularité d'être "sous-critiques ", c'est-à-dire que la réaction en chaine ne peut ni démarrer ni s'entretenir spontanément sans l'apport de neutrons externes. Dans la plupart des concepts, un accélérateur fournit des protons de haute énergie, qui vont frapper une cible faite d'un métal lourd (par exemple du plomb) située au cœur du réacteur. Cela produit des réactions nucléaires qui libèrent un grand nombre de neutrons. Ceux-ci vont créer des fissions dans le cœur et ainsi générer une certaine puissance qui pourra devenir nulle sur commande, par l'arrêt de l'accélérateur. Ce contrôle de la puissance par l'accélérateur permet d'utiliser des combustibles un peu exotiques comme les actinides mineurs ${ }^{(b)}$ dont les propriétés ne permettent pas l'usage en réacteur critique. L'ADS est alors exploité en version rapide, avec du plomb liquide comme modérateur-caloporteur. Le principal défi à relever pour la mise en œuvre de ces systèmes (en dehors de la chimie du plomb liquide, point commun avec le LFR) est d'atteindre un niveau de fiabilité $\mathrm{du}$ faisceau de particules encore jamais atteint. Ces réacteurs sont étudiés depuis plus de vingt ans, en particulier en Europe et en France, avec une intense R\&D sur les accélérateurs linéaires. La construction en Belgique d'un démonstrateur d'environ 100 MW, MYRRHA, est désormais programmée en deux étapes : une pour l'accélérateur en 2026 et l'autre pour le réacteur en 2033.

\section{Conclusion}

Les recherches sur de nouveaux systèmes de production d'énergie nucléaire ne se limitent pas aujourd'hui à celles réalisées dans le cadre du GIF, dont l'initiative a eu le mérite de fixer des critères d'évolution des concepts toujours plus exigeants, notamment vis-à-vis de la sureté nucléaire et de la question des déchets, et de relancer une R\&D concertée entre les pays ayant une industrie nucléaire. On peut apprécier que les réticences les plus fortes exprimées par la société envers le nucléaire aient été d'une certaine manière, prises en compte par la recherche. Cependant, développer de nouveaux réacteurs jusqu'au niveau industriel face aux progrès réalisés par les autres sources d'énergie, notamment renouvelables, ne peut s'affranchir des aspects économiques. C'est pourquoi, en dehors de ces nouveaux concepts, de gros efforts sont également mis, notamment par l'industrie française, sur l'étude de réacteurs de troisième génération basés sur l'optimisation des réacteurs à eau actuels. En résumé, les options pour l'avenir du nucléaire sont nombreuses. Mais l'effort de recherche, qui doit être anticipé sur des décennies, ne peut être mené à force égale sur chaque système. L'enjeu est de mener une R\&D pouvant aboutir à un système industriel sans s'interdire d'explorer des voies plus audacieuses pour le futur.

\section{En savoir plus}

- Les réacteurs nucléaires à caloporteur sodium, CEA, Monographie de la DEN, Éditions Le Moniteur (2014)

- Les réacteurs nucléaires à caloporteur gaz, CEA, Monographie de la DEN, Éditions Le Moniteur (2006).

- "Technology Roadmap Update for Generation IV Nuclear Energy Systems", Gen. IV International Forum, January 2014, NEA/OCDE.

- Gen. IV International Forum, www.gen-4.org/gif/jcms/c_9260/public

- "Vers un cycle du combustible nucléaire durable : Évolution et tendances », NEA/ OCDE (2012) N6981. www.oecd-nea. org/ndd/reports/2012/6981-trendssustainability-fuel-cycle-fr.pdf

- "Perspective on the use of Thorium in the Nuclear Fuel Cycle", NEA/OCDE (2015) $\mathrm{N}^{\circ}$ 7224. www.oecd-nea.org/ science/pubs/2015/7224-thorium.pdf

a. Un noyau d'uranium 238 qui capture un neutron donne un noyau d'uranium 239, noyau radioactif, qui se désintègre en un noyau de plutonium 239.

b. Noyaux lourds très radiotoxiques créés par captures neutroniques dans les réacteurs. 